\title{
A self-setting iPSMSC-alginate-calcium phosphate paste for bone tissue engineering
}

\author{
Ping Wang ${ }^{1,2}$, Yang Song ${ }^{1}$, Michael D. Weir ${ }^{1}$, Jinyu Sun ${ }^{1}$, Liang Zhao ${ }^{1,3}$, \\ Carl G. Simon ${ }^{4}$, Hockin H. K. Xu ${ }^{1,5-7}$ \\ ${ }^{1}$ Biomaterials \& Tissue Engineering Division, Department of Endodontics, Prosthodontics and \\ Operative Dentistry, University of Maryland Dental School, Baltimore, MD 21201, USA \\ ${ }^{2}$ State Key Laboratory of Oral Diseases, West China Hospital of Stomatology, Sichuan \\ University, Chengdu, Sichuan 610041, China \\ ${ }^{3}$ Department of Orthopaedic Surgery, Nanfang Hospital, Southern Medical University, \\ Guangzhou, Guangdong 510515, China \\ ${ }^{4}$ Polymers Division, National Institute of Standards \& Technology, 100 Bureau Drive, \\ Gaithersburg, MD, 20899, USA \\ ${ }^{5}$ Center for Stem Cell Biology and Regenerative Medicine, University of Maryland School of \\ Medicine, Baltimore, MD 21201, USA \\ ${ }^{6}$ University of Maryland Marlene and Stewart Greenebaum Cancer Center, University of \\ Maryland School of Medicine, Baltimore, MD 21201, USA \\ ${ }^{7}$ Mechanical Engineering Department, University of Maryland Baltimore County, Baltimore, \\ MD 21250, USA
}

For: Dental Materials

(Submitted in March 2015; revised and resubmitted in September 2015)

\section{Correspondence:}

Dr. Hockin H. K. Xu, Professor, Director of Biomaterials \& Tissue Engineering Division, Department of Endodontics, Prosthodontics and Operative Dentistry, University of Maryland Dental School, Baltimore, MD 21201 (Email: hxu@umaryland.edu); Dr. Liang Zhao, Department of Orthopaedic Surgery, Nanfang Hospital, Southern Medical University, Guangzhou, Guangdong 510515, China (Email: lzhaonf@126.com).

Short title: iPSMSC-alginate-calcium phosphate paste for bone engineering

Keywords: bone tissue engineering, calcium phosphate cement, human induced pluripotent stem cells, alginate microbeads, injectable, animal studies. 


\section{ABSTRACT}

Objectives. Calcium phosphate cements (CPCs) are promising for dental and craniofacial repairs. The objectives of this study were to: (1) develop an injectable cell delivery system based on encapsulation of induced pluripotent stem cell-derived mesenchymal stem cells (iPSMSCs) in microbeads; (2) develop a novel tissue engineered construct by dispersing iPSMSC-microbeads in CPC to investigate bone regeneration in an animal model for the first time.

Methods. iPSMSCs were pre-osteoinduced for 2 weeks (OS-iPSMSCs), or transduced with bone morphogenetic protein-2 (BMP2-iPSMSCs). Cells were encapsulated in fast-degradable alginate microbeads. Microbeads were mixed with CPC paste and filled into cranial defects in nude rats. Four groups were tested: (1) CPC-microbeads without cells (CPC control); (2) CPCmicrobeads-iPSMSCs (CPC-iPSMSCs); (3) CPC-microbeads-OS-iPSMSCs (CPC-OSiPSMSCs); (4) CPC-microbeads-BMP2-iPSMSCs (CPC-BMP2-iPSMSCs).

Results. Cells maintained good viability inside microbeads after injection. The microbeads were able to release the cells which had more than 10-fold increase in live cell density from 1 to 14 days. The cells exhibited up-regulation of osteogenic markers and deposition of minerals. In vivo, new bone area fraction (mean $\pm \mathrm{SD} ; \mathrm{n}=5$ ) for CPC-iPSMSCs group was $(22.5 \pm 7.6) \%$. New bone area fractions were $(38.9 \pm 18.4) \%$ and $(44.7 \pm 22.8) \%$ for CPC-OS-iPSMSCs group and CPC-BMP2-iPSMSCs group, respectively, $2-3$ times the $(15.6 \pm 11.2) \%$ in CPC control at 12 weeks ( $\mathrm{p}<0.05)$. Cell-CPC constructs accelerated scaffold resorption, with CPC-BMP2iPSMSCs having remaining scaffold material that was 7-fold less than CPC control.

Significance. Novel injectable CPC-microbead-cell constructs promoted bone regeneration, with OS-iPSMSCs and BMP2-iPSMSCs having 2-3 fold the new bone of CPC control. Cell delivery accelerated scaffold resorption, with CPC-BMP2-iPSMSC having remaining scaffold material that was 7-fold less than CPC control. Therefore, CPC-microbead-iPSMSC is a promising injectable material for orthopedic, dental and craniofacial bone regenerations. 


\section{Introduction}

The generation of induced pluripotent stem cells (iPSCs) is an exciting discovery in the field of cell-based therapy [1]. Through expression of a small combination of transcription factors, somatic cells can be converted into an embryonic state, thus, exhibiting tremendous possibilities in treatment of various diseases. IPSCs represent an enormous source of patientspecific stem cells derived from plentiful and easily accessible tissues like skin, hair and fat, etc. Development of virus-free and vector-free reprogramming technologies reduces the chance of virally-induced tumor formation, thus provides optimism for clinical applications of iPSCs [2-3]. When transplanted back into the patients, iPSCs need to be induced into high-quality progenitor cells like mesenchymal stem cells (MSCs), or fully-differentiated homogenous mature cells to circumvent the risk of teratoma formation caused by undifferentiated cells contaminating the final products [4-5]. It has been reported that iPSC-derived MSCs (iPSMSCs) exhibited a higher proliferative capability than bone marrow MSCs (BMSCs) [6], and are less tumorigenic than undifferentiated iPSCs and BMSCs [7-9]. Thus in the field of bone tissue engineering, iPSMSCs escalate the hope especially for patients with compromised health conditions whose autologous BMSCs are no longer vibrant for tissue repair and regeneration [10].

Calcium phosphate cements are promising bone substitutes with excellent bioactivity, biocompatibility and osteoconductivity [11-14]. These materials stand out as injectable bone cements owing to their self-setting and in situ-hardening capabilities [15-17]. One such cement consisted of tetracalcium phosphate [TTCP: $\mathrm{Ca}_{4}\left(\mathrm{PO}_{4}\right)_{2} \mathrm{O}$ ] and dicalcium phosphate (DCPA: $\mathrm{CaHPO}_{4}$ ), and was referred to as CPC [17]. The CPC powder can be mixed with an aqueous liquid to form a paste that can be injected or sculpted during surgery to conform to the defects in hard tissues. CPC was approved in 1996 by the Food and Drug Administration for repairing 
craniofacial defects in humans, thus becoming the first CPC available for clinical use [18].

Our previous studies enhanced the mechanical, physical and biological properties of CPC through the introduction of absorbable fibers [19], chitosan [20], mannitol porogen [21], gasfoaming agents [22], alginate microbeads [23], and biofunctionalization [24]. These approaches improved the CPC's mechanical strength, setting time, degradability, macroporosity, cell attachment, and delivery of cells and growth factors. Potential dental and craniofacial applications of an improved CPC include periodontal bone lesion repair, socket preservation, maxillary sinus floor elevation, augmentation of deficient implant sites, ridge augmentation, as well as other dental and orthopedic applications [17,18].

Our recent study showed that osteoinduced iPSMSCs seeded on pre-formed CPC scaffolds in rat cranial defects had comparable in vivo bone regeneration capability to BMSCs and umbilical cord MSCs (UCMSCs), but significantly higher than CPC control [25]. No teratoma was found during a 12 week observation [25]. Although it was promising in supporting iPSMSCs' role in prompting bone regeneration efficiency of CPCs, cells were only loaded on one side of the scaffolds. This type of static cell seeding method has limitations of low seeding efficiency and minimal cell penetration into scaffold, leading to non-uniform distribution of cells and subsequently compromised regeneration in vivo [26]. To address these problems, in the present study, alginate microbeads were used as cell delivery vehicles to protect the encapsulated cells during CPC paste mixing, injection and setting reactions. The $\mathrm{CPC}$-microbead-constructs can be readily injected or placed into bone defects with minimal invasion and intimate adaptation to complex defect shapes [27]. Alginate has been selected because it is non-cytotoxic and can form an ionically cross-linked network under mild conditions producing no detrimental effects to cells [28]. To promote alginate degradation and subsequent cell release, fast-degradable alginate- 
fibrin microbeads were fabricated following a previous study [29]. Furthermore, to enhance osteogenicity, iPSMSCs were either pre-osteoinduced for 2 weeks (OS-iPSMSCs), or transduced with bone morphogenic protein-2 (BMP2) gene (BMP2-iPSMSCs).

The aims of this study were to: (1) develop a novel injectable cell delivery system based on iPSMSC encapsulation in alginate microbeads and investigate cell viability, proliferation and osteogenic differentiation; and (2) develop a novel tissue engineered construct by dispersing iPSMSC-microbeads in CPC and investigate bone regeneration in vivo. The following hypotheses were tested: (1) microencapsulation and injection would not harm the encapsulated iPSMSCs; (2) iPSMSCs released from fast-degradable microbeads could proliferate and differentiate into osteogenic lineage; (3) cell-encapsulating-microbeads can induce osteogenic differentiation of co-cultured BMSCs; and (4) pre-osteogenic differentiation and BMP2 transduction would promote bone regeneration of iPSMSCs in CPC constructs in vivo.

\section{Materials and methods}

\subsection{Derivation of MSCs from iPSCs}

Human iPSC BC1 line was maintained on mitotically-inactivated murine embryonic fibroblasts (MEF) feeder [30]. IPSCs were detached from MEF and dissociated into clumps by treatment with collagenase type IV. The dissociated iPSC clumps were collected, resuspended, and transferred to ultra-low attachment cell culture flasks (Corning, Corning, NY). After 10 days (d), EBs were transferred onto $0.1 \%$ gelatin coated culture dishes. Cells growing from EBs were cultured and upon $70 \%$ confluence, outgrowth cells were selectively isolated using cell scrapers and sub-cultured in MSC growth media, which consisted of low glucose Dulbecco's modified Eagle's media (Gibco, Grand Island, NY) supplemented with 10\% fetal bovine serum (HyClone, 
Logan, UT), $100 \mathrm{U} / \mathrm{mL}$ penicillin and $100 \mathrm{mg} / \mathrm{mL}$ streptomycin (Gibco). The differentiated cells were passaged until a homogeneous fibroblastic morphology appeared and were termed iPSMSCs. Our previous study confirmed that iPSMSCs generated from this method expressed surface markers of MSCs (CD29, CD44, CD166, CD73), and were negative for typical hematopoietic (CD34), endothelial (CD31) and pluripotent markers (TRA-1-81 and OCT 3/4). The iPSMSCs could differentiate into three characteristic mesenchymal lineages including osteoblasts, adipocytes and chondrocytes [31]. The iPSMSCs used in this study have also been tested positive for CD29, CD44, CD166, CD73, but negative for CD34, CD31, TRA-1-81 and OCT 3/4. The 3-5th passage iPSMSCs were used in the following experiments.

\subsection{BMP2 gene transduction of iPSMSCs}

Lentiviruses with human BMP2 (GenTarget, San Diego, CA) were used for gene transduction. Passage 3 iPSMSCs were exposed to 15 multiplicity of infection of BMP2 lentiviruses for $3 \mathrm{~d}$. These cells are referred to as BMP2-iPSMSCs. Our previous study showed that transduction efficacy was more than $68.8 \%$, and BMP2 gene and protein were stably expressed from passage 5 to 8 . Cell proliferation was comparable to iPSMSCs while osteogenic differentiation was enhanced in BMP2-iPSMSCs [32].

\subsection{Synthesis of alginate microbeads with cell encapsulation}

Alginate (64\% guluronic acid, MW = 75,000-220,000g/mol, ProNova, Oslo, Norway) was oxidized at $7.5 \%$ oxidation to increase its degradability [29]. The oxidized alginate was dissolved in saline at a concentration of $1.2 \%$. Fibrinogen from bovine plasma (Sigma) was added at a concentration of $0.1 \%$ to the alginate solution to further accelerate alginate 
degradation [29]. Cells were added to the alginate-fibrinogen solution at a density of $1 \times 10^{6}$ cells $/ \mathrm{mL}$. The cell solution was loaded into a syringe which was connected to a bead generating device (Var J1, Nisco, Zurich, Switzerland). Nitrogen gas at a pressure of 8 psi was established to form a coaxial air flow to break up alginate droplets. The droplets fell into a well containing 125 $\mathrm{mL}$ of $100 \mathrm{mmol} / \mathrm{L}$ calcium chloride plus $125 \mathrm{NIH}$ units of thrombin (Sigma). Calcium chloride caused alginate to crosslink, while the reaction between fibrinogen and thrombin produced fibrin. This yielded cell-encapsulating-alginate-fibrin-microbeads with diameters of about 100-500 $\mu \mathrm{m}$, with a mean of $311 \mu \mathrm{m}$ [29]. Three groups of cell-encapsulating-microbeads were produced. (1) iPSMSCs. (2) Pre-osteoinduced iPSMSCs (OS-iPSMSCs). These were cells that underwent $2 \mathrm{w}$ osteogenic differentiation in osteogenic media (OS media) which consisted of MSC growth media supplemented with $100 \mathrm{nM}$ dexamethasone, $10 \mathrm{mM}$ ß-glycerophosphate, $0.05 \mathrm{mM}$ ascorbic acid, and $10 \mathrm{nM} \mathrm{1a,25-dihydroxyvitaminD3} \mathrm{(Sigma).} \mathrm{(3)} \mathrm{BMP2-iPSMSCs.}$

\subsection{Cell viability and proliferation}

Cell-encapsulating-microbeads ( $50 \mu \mathrm{L})$ were added to each well of 24 well-plates with 1 mL MSC growth media. Twelve wells were prepared at each time-point (1, 3, 7 and $14 \mathrm{~d})$, with six wells for live/dead staining, and six wells for cell proliferation assay. Cells were stained with a live/dead kit (Invitrogen) and observed via epifluorescence microscopy (Eclipse TE-2000S, Nikon, Melville, NY). Two images were taken at random locations for each sample, with 6 samples yielding 12 images for iPSMSC-microbeads at each time point. Live and dead cells were counted separately in green or red channels via Image J2 software. After subtracting the background with an automatic threshold, the images were converted to binary images. Objects in a binary image that overlapped each other were corrected by using the watershed separation 
function of the software. Once the image has been segmented, the menu command "Analyze/Analyze particles" were used to obtain various information regarding particle size and numbers. The percentage of live cells $\mathrm{P}=$ number of live cells / (number of live cells + number of dead cells) x 100\%. Live cell density D = number of live cells in the image / image area [29].

Cell counting kit (CCK-8; Dojindo, Tokyo, Japan) was used to evaluate cell proliferation

from 1 to $14 \mathrm{~d}$. To investigate if the injection process would harm the encapsulated cells, iPSMSC viability at $1 \mathrm{~d}$ was compared between: (1) cells in microbeads without injection, and (2) cells in microbeads after injection from a $10 \mathrm{~mL}$ syringe (Free-Flo, Kerr, Romulus, MI) with a tip opening of $2.7 \mathrm{~mm}$ which was similar to the inner diameter of a 10-gauge needle [27].

\subsection{Osteogenic differentiation and mineralization of cell-encapsulating-microbeads}

Three types of microbeads were cultured in OS media for 2 weeks. At 1, 7 and $14 \mathrm{~d}$, TaqMan gene expression kits were used to quantify targeted genes on human alkaline phosphatase (ALP, Hs00758162_ml, RefSeq NM_000478.4, assay location: 1120, amplicon length: 84; RefSeq NM_001127501.2, assay location: 955, amplicon length: 84; RefSeq NM_001177520.1, assay location: 839, amplicon length: 84), Runt-related transcription factor (RUNX2, Hs00231692_ml, RefSeq NM_001015051.3, assay location: 900, amplicon length: 116; RefSeq NM_001024630.3, assay location: 900, amplicon length: 116; RefSeq NM_001278478.1, assay location: 648, amplicon length: 116), collagen type-I, alpha 1 (COL1A1, Hs00164004_m1, RefSeq NM_000088.3, assay location: 230, amplicon length: 66), osteocalcin (OCN, Hs01587814_g1, RefSeq NM_199173.4, assay location 177, amplicon length: 116), and glyceraldehyde 3-phosphate dehydrogenase (GAPDH, Hs99999905, RefSeq NM_002046.4, assay location: 229, amplicon length: 122). Relative expression was evaluated 
using the $2^{-\Delta \Delta C t}$ method and normalized by the $C_{t}$ of the housekeeping gene GAPDH. $C_{t}$ of iPSMSCs in growth media cultured on tissue culture polystyrene (TCPS) for $1 \mathrm{~d}$ served as the calibrator $[29,32]$.

At 1, 7 and 14 d, cell-encapsulating-microbeads were stained with Alizarin Red S (ARS, Millipore, Billerica, MA). The ARS concentration was measured by the Osteogenesis Quantitation Kit (Millipore) [29,32].

\subsection{Osteogenic differentiation of BMSCs in co-culture with cell-encapsulating-microbeads}

Co-culture of cell-encapsulating-microbeads and non-encapsulated BMSCs was conducted in a transwell system (BD Biosciences), which allowed the sharing of conditioned media without cell-cell contact. Non-encapsulated BMSCs were seeded into the lower compartment of 12 -well plates at $5 \times 10^{4}$ cells/well, and $50 \mu \mathrm{L}$ cell-encapsulating-microbeads were added to the upper compartments for co-culture in MSC growth media for 2 weeks. At 1, 7 and $14 \mathrm{~d}$, TaqMan gene expression kits were used to quantify targeted genes on human ALP (Hs00758162_ml), RUNX2 (Hs00231692_ml), OCN (Hs01587814_g1), osteopontin (OPN, Hs00959010_m1, RefSeq NM_000582.2, assay location: 657, amplicon length: 84; RefSeq NM_001040058.1, assay location: 699, amplicon length: 84; RefSeq NM_001040060.1, assay location: 618, amplicon length: 84; RefSeq NM_001251829.1, assay location: 576, amplicon length: 84; RefSeq NM_001251830.1, assay location: 888, amplicon length: 84) and GAPDH (Hs99999905). Gene expression was evaluated using the $2^{-\Delta \Delta C t}$ method as described above.

\subsection{Fabrication of CPC scaffolds}

CPC powder was prepared following a previous study [33-34]. Briefly, TTCP 
$\left[\mathrm{Ca}_{4}\left(\mathrm{PO}_{4}\right)_{2} \mathrm{O}\right]$ was synthesized using DCPA $\left(\mathrm{CaHPO}_{4}\right)$ and calcium carbonate (both from J.T. Baker, Philipsburg, NJ) which were mixed and heated at $1500{ }^{\circ} \mathrm{C}$ for 6 hours (h) in a furnace (Model 51333, Lindberg, Watertown, WI). The heated mixture was quenched to room temperature in a desicator, ground in a ball mill (Retsch PM4, Brinkman, NY) and sieved to obtain TTCP powder with a median particle size of $5 \mu \mathrm{m}$. The commercial DCPA powder was ground for $24 \mathrm{~h}$ in the ball mill in $95 \%$ ethanol and sieved to obtain a median particle size of approximately $1 \mu \mathrm{m}$. Then the TTCP and DCPA powders at 1:3 molar ratio were thoroughly mixed in a micromill (Bel-Alert Products, Pequannock, NJ) to form the CPC powder [33-34]. The microbeads were incorporated into CPC at $50 \%$ by volume. Degradable suture fibers (Ethicon, Somerville, $\mathrm{NJ}$ ) were cut into $3 \mathrm{~mm}$ filaments and added into $\mathrm{CPC}$ at $10 \%$ by volume for mechanical reinforcement. The $\mathrm{CPC}$ liquid was $0.2 \mathrm{M} \mathrm{Na}_{2} \mathrm{HPO}_{4}$. A powder: liquid ratio of 2:1 was used to form a flowable CPC paste.

\subsection{In vivo double cranial bone defects in rats}

Double cranial defects of $5 \mathrm{~mm}$ each were created in $8 \mathrm{w}$-old male athymic nude rats (Hsd:RH-Fox $1^{\mathrm{mu}}, 200-250 \mathrm{~g}$, Harlan, Indianapolis, IN) following a protocol approved by the University of Maryland Baltimore (IACUC \# 0909014) and NIH animal-care guidelines. Briefly, under general anesthesia, two full-thickness $5 \mathrm{~mm}$ defects were made in the calvarium under continuous saline irrigation [35]. CPC paste was mixed at the time of implantation and set in situ to fill the bone defects. Four groups were tested: (1) CPC-microbeads without cells (CPC control); (2) CPC-microbeads-iPSMSCs (CPC-iPSMSCs); (3) CPC-microbeads-OS-iPSMSCs (CPC-OS-iPSMSCs); (4) CPC-microbeads-BMP2-iPSMSCs (CPC-BMP2-iPSMSCs). Grafts were harvested after 12 weeks $(n=5)$. 


\subsection{Histomorphometric analyses}

Specimens were decalcified and embedded in paraffin. The central part of the implant and defect was cut into $5 \mu \mathrm{m}$-thick sections and stained with hematoxylin and eosin (HE) and Masson's Trichrome (MT). New bone area, residual material area and total defect area was measured within the boundaries of defects in each section by Image Pro Plus Software (Media Cybernetics, Carlsbad, CA). New bone area fraction (NBAF) was calculated as the new bone area divided by total defect area $(n=5)$. CPC scaffold residual material area fraction (RMAF) was calculated as the residual scaffold material area divided by total defect area $(n=5)$.

\subsection{Identification of encapsulated cells by immunohistochemistry (IHC)}

Human origin of engineered bone constructs following in vivo implantation was detected using mouse monoclonal anti-human nuclei antibodies (Millipore). Tissue sections were deparaffinized with xylene, and rehydrated with a graded series of ethanol washes. The epitopes were recovered by incubation in citrate buffer at $70^{\circ} \mathrm{C}$ for $40 \mathrm{~min}$, and the endogenous peroxidase activity was blocked with $3 \% \mathrm{H}_{2} \mathrm{O}_{2}$. The slides were then blocked with $1 \%$ BSA for 30 min to suppress nonspecific staining and stained with primary antibodies $(1: 50)$ overnight in a humidified environment. The specimens were subsequently incubated with secondary antibody against mouse $\operatorname{IgG}(1: 500)$ for $30 \mathrm{~min}$ at $37^{\circ} \mathrm{C}$. Incubation was followed by streptavidin-HRP and diaminobenzidine (DAB) substrate, and counterstaining with hematoxylin solution. Negative controls were performed following the same procedures but without primary antibody incubation.

\subsection{Statistical analyses}


Statistical analyses were performed using Statistical Package for the Social Sciences

(SPSS 19.0, Chicago, IL). All data were expressed as the mean value \pm standard deviation (SD). All in vitro tests were independently repeated three times, with at least triplicate cultures for each condition. Statistical significance was analyzed by using the one-way analyses of variance (ANOVA) and Student-Newman-Keuls test. A confidence level of 95\% $(\mathrm{p}<0.05)$ was considered significant.

\section{Results}

The viability of iPSMSCs in microbeads was not adversely affected by the microencapsulation procedure as shown by live/dead staining (Fig.1 E-H). The live cell percentage at $1 \mathrm{~d}$ was around $90.7 \%$ (Fig.1 I). Live cells appeared as green dots dispersed in microbeads, with a few dead cells (red dots) (Fig.1 E). At 3 d, some cells released from microbeads exhibiting a flat and polygonal morphology (Fig.1 B, F). From 7 to $14 \mathrm{~d}$, as more cells were released, cell proliferation was significantly enhanced, and live cell percentage was over $95 \%$. Over 10 -fold increase in live cell density occurred from 1 to $14 \mathrm{~d}$. CCK-8 assay showed increased OD values, indicating cell proliferation over time (Fig.1 L). There was no significant difference in cell viability between the no injection group and the after injection group, indicating that the CPC injection process did not harm the encapsulated cells.

Gene expressions of osteogenic markers of the encapsulated cells were plotted in Fig.2. The expressions of early and middle stage osteogenic markers ALP, RUNX2 and COL1A1 of OS-iPSMSCs were peaked at $1 \mathrm{~d}$, while the late stage marker OCN was dramatically increased at 7 d. Up-regulations of ALP, RUNX2, COL1A1 and OCN were observed in BMP2-iPSMSCs at 7 $\mathrm{d}$, while these expressions in iPSMSC-microbeads were continuously elevated from 1 to $14 \mathrm{~d}$. In 
general, OS-iPSMSC and BMP2-iPSMSC-microbeads groups demonstrated an earlier upregulation of osteogenic genes than the iPSMSC-microbeads group, indicating an advanced osteogenic status in these two groups. Nevertheless, iPSMSCs encapsulated in micrbeads did undergo osteogenic differentiation upon induction. These results showed that the microencapsulation did not exert any harmful effects on the differentiation ability of the encapsulated cells. ARS staining of the synthesized bone mineral matrix became denser and darker over time, consistent with the osteogenesis assay (Fig.2 F). Minerals synthesized by cells increased dramatically at $14 \mathrm{~d}(\mathrm{p}<0.05)$. Mineral concentration was significantly higher in OSiPSMSCs and BMP2-iPSMSCs, compared to iPSMSCs at $14 \mathrm{~d}$.

Up-regulations of ALP, RUNX2, OCN and OPN were observed in BMSCs when cocultured with cell-encapsulating-microbeads in the absence of OS media (Fig.3). The osteogenic influence of iPSMSC-microbeads on the co-cultured BMSCs was at levels comparable to, or even higher than, that of OS-iPSMSC-micorbeads and BMP2-iPSMSC-microbeads.

When implanted into nude rats, no signs of inflammation or teratoma formation were observed in any samples. Histological staining showed that 2 out of 5 samples in both CPC-OSiPSMSCs and CPC-BMP2-iPSMSCs groups showed nearly complete osseous bridge of the defects on the dura side (Fig.4). In CPC control, new bone was mainly observed in the junction area between the scaffold and native bone. In undifferentiated iPSMSC-constructs, a limited amount of new bone was also found in the center of defects. In sharp contrast, groups with differentiated OS-iPSMSCs and BMP2-iPSMSCs showed much more new bone, spanning the central and marginal regions of the defects. Higher magnification showed new bone with a typical organized and mature bone morphology (Fig.4 I, J). As shown in Fig.4 I, a layer of osteoblasts lined the boundaries of new bone. Osteocytes and new blood vessels were surrounded 
by a matrix of woven bone. Osteoclast-like multinuclear giant cells were found in the newly formed tissues, indicating an active process of scaffold material degradation and new bone formation. Masson's staining (Fig.4 J) showed uncalcified collagen fibers intermingled between the new bone and the scaffold.

IHC staining for human nuclear antigen was shown in Fig.5. Positive staining was mainly found within non-mineralized fibrous tissue in the defects in all cell-seeded groups, with a few positive cells embedded in the mineralized new bone. No positive staining was found in control. These results confirmed the direct participation of the delivered human cells in bone regeneration.

In Fig. 5E, new bone area fraction at 12 weeks was $(22.5 \pm 7.6) \%,(38.9 \pm 18.4) \%$ and $(44.7 \pm 22.8) \%$ in CPC-iPSMSCs, CPC-OS-iPSMSCs and CPC-BMP2-iPSMSCs, respectively. Hence, CPC-OS-iPSMSCs and CPC-BMP2-iPSMSCs had new bone that was 2-3 fold the (15.6 $\pm 11.2) \%$ for CPC control ( $\mathrm{p}<0.05)$. In Fig. 5F, the remaining CPC material was $(26.5 \pm 8.5) \%$, $(17.0 \pm 4.2) \%$ and $(6.9 \pm 2.5) \%$ in CPC-iPSMSCs, CPC-OS-iPSMSCs and CPC-BMP2iPSMSCs, respectively. Therefore, cell seeding increased the CPC resorption rate in vivo, and the CPC-BMP2-iPSMSC defects had remaining scaffold material that was 7-fold less than the $(53.6 \pm 3.5) \%$ for CPC control $(\mathrm{p}<0.05)$

\section{Discussion}

Cell therapy has the potential to greatly improve current methods of bone regeneration through increased bioactivity of scaffolds and localized growth factor delivery [36-37]. MSCs are the focus of many cell-based tissue-engineering therapies [38-39]. IPSMSCs are an important alternative source for the mass production of MSCs. A majority of studies induce iPSCs to differentiate into MSCs prior to initiating osteogenic differentiation [31, 40-44]. IPSMSCs have 
greater capacity of cell proliferation than BMSCs (over 10 fold higher in telomerase activity) and are able to proliferate for 120 population doublings without losing self-renewal capacity and MSC characteristics [6]. In addition, iPSMSCs could reduce the chance of tumor formation associated with iPSCs and BMSCs [7-9]. There are various protocols for deriving MSCs from iPSCs. The present study adopted a classical protocol for differentiating embryonic stem cells (ESCs) into MSCs, which involved EB formation [45]. After transferring EBs onto gelatincoated plates, an outgrowth of epithelial phenotype cells was derived via migrating out of the EBs. These cells then underwent epithelial-to-mesenchymal transition (EMT) to acquire the mesenchymal phenotype [46]. Although this method is still the mainstream in iPSMSC generation, efforts to find short-cuts have never ceased. Non-EB approaches have been promoted when researchers find that EB formation is not absolutely necessary [47]. MSCs can be directly derived from iPSCs via co-culture systems or conditioned media from osteogenic cells [41], directly plating cells in MSC growth media [42-43], adding additional small molecules or growth factors [40, 48], or seeding cells onto osteogenic scaffolds [44, 49]. All these methods succeed in yielding iPSMSCs which can differentiate down the osteoblastic lineage, presenting ALP activity, osteogenic gene expression and mineral synthesis. In vivo studies demonstrate de novo bone formation or mineral deposition in iPSC-implanted scaffolds and direct involvement of transplanted cells in bone regeneration $[8,42,49-50]$. However, it remains unclear which protocol leads to the most efficient osteogenic differentiation.

Cell delivery methods are important in attainment of functional properties of tissue engineered constructs and subsequent tissue development [26, 51]. A common method is static loading of a concentrated cell suspension onto the surface of a scaffold. This technique has several limitations including low seeding efficiency and minimal cell penetration of scaffold 
walls [26]. On the other hand, it's not feasible to directly seed and mix cells into CPC paste because ionic exchanges and $\mathrm{pH}$ variations during CPC setting would damage the cells [52]. Once CPC is set, it is biocompatible and not cytotoxic. Therefore, the present study used a microencapsulation technology as cell carriers to release the cells only after the CPC scaffold had set. Live/dead and CCK-8 assays showed that after encapsulation and injection, the encapsulated iPSMSCs maintained good viability. The encapsulated cells were released at $3 \mathrm{~d}$, and the released cells had fast proliferation and successful osteogenic differentiation. The OSiPSMSC and BMP2-iPSMSC-microbeads groups demonstrated an earlier upregulation of osteogenic genes and a greater amount of mineral deposition during osteoinduction than the iPSMSC-microbeads group. However, the osteogenic effect exerted by the iPSMSC-microbeads constructs on the co-cultured BMSCs was comparable to the osteogenic effects by OS-iPSMSC and BMP2-iPSMSC-microbeads. These results suggest that the iPSMSCs, OS-iPSMSCs and BMP2-iPSMSCs all released bioactive factors that acted on a paracrine basis to induce the osteogenic differentiation of the co-cultured BMSCs. Further studies are needed to identify the specific factors released by these cells to induce the osteogenic differentiation.

One disadvantage of alginate microbeads is their weak mechanical strength, making them incapable to withstand pressure in load-bearing sites or to maintain the shape integrity [53]. In the present study, the novel construct containing iPSMSC-microbeads in CPC paste with fibers for mechanical reinforcement would have load-bearing capability after injection and CPC setting [27]. Our previous studies showed that the incorporation of fibers increased the strength and toughness for the CPC-microbead construct, without compromising its injectability [27]. When microbeads were mixed with CPC, homogenous cell distribution throughout the entire scaffold could be achieved. During the CPC setting and injection process, microbeads acted as protection 
shells for the encapsulated cells, and as provisional matrix to maintain the regenerative space [21, 27]. After degradation of the fast-resorbable microbeads, cells could be released and macropores were formed within the scaffold [22]. These pores with sufficient dimensions provided pathways for cell migration and proliferation, ingrowth of blood vessels and new bone, and facilitate diffusion of oxygen and nutrients [54]. Meanwhile, the CPC scaffold provided a biocompatible substrate for cell attachment, proliferation and differentiation.

At 12 weeks in vivo, CPC control showed primarily large blocks of residual CPC material and loose connective tissues in the defects with small amounts of new bone at the margins. In contrast, CPC-OS-iPSMSCs and CPC-BMP2-iPSMSCs had more new bone than CPC-iPSMSC and CPC control groups $(\mathrm{p}<0.05)$. The cell-encapsulating constructs had less residual CPC than control $(\mathrm{p}<0.05)$. The following are possible mechanisms for these results. Initially, the released cells from microbeads directly contributed to tissue regeneration as evidenced by IHC identification of human originated cells. Then, via a cell-mediated paracrine stimulation, the native cells including osteoblast progenitors and multinucleated giant cells from the host were responsible for subsequent bone formation and remodeling [8, 50]. Meanwhile, extracellular matrix secreted by implanted cells was acting to facilitate migration and adhesion of the recruited cells to the material surface [55]. In addition, the stimulated host cells such as osteoblasts could produce factors such as granulocyte-macrophage colony-stimulating factor (GM-CSF), macrophage colony-stimulating factor (M-CSF) which were of vital importance to the migration and proliferation of osteoclast progenitor cells [56]. Further studies are needed to investigate these aspects in vivo in more detail.

The injectable CPC-stem cell paste may be promising for various applications. For sintered hydroxyapatite and other bioceramics to fit into a bone cavity, the surgeon needs to 
machine the graft to the desired shape or carve the surgical site around the implant, which leads to increases in bone loss, trauma and surgical time. The injectable CPC-stem cell paste can be molded and set in situ to provide intimate adaptation to the contours of defect surfaces, and can be shaped for esthetics in dental and craniofacial reconstructions. In addition, to seed stem cells into the deep interior of a pre-fabricated hydroxyapatite or bioceramic scaffold is difficult, with most cells on the scaffold surface. In contrast, the injectable CPC-stem cell paste can have cells throughout the entire construct volume. The injectable and in situ setting iPSMSC-microbeadCPC paste could be useful for mandibular and maxillary ridge augmentation, for major reconstructions of the maxilla or mandible after trauma or tumor resection, and for supporting metal dental implants or augmentation of deficient implant sites. As demonstrated in the present study, the iPSMSC-microbead-CPC constructs could substantially increase new bone regeneration and scaffold resorption rate in vivo, compared to CPC control without cell deliver. Further studies are needed to optimize CPC composition and porosity as well as cell seeding and induction parameters for even more rapid bone regeneration in vivo.

\section{Conclusions}

In conclusion, we advanced our previous findings on iPSMSCs' osteogenic capability and fastdegradable alginate-fibrin microbeads to construct a novel injectable tissue engineered bone by dispersing iPSMSC-microbeads in CPC paste. This represents the first report on the reconstruction of cranial bone defects in vivo using microencapsulated iPSMSC-CPC constructs. The constructs significantly enhanced bone regeneration especially via OS-iPSMSCs and BMP2iPSMSCs in vivo. OS-iPSMSCs and BMP2-iPSMSCs achieved 2-3 fold increases in bone regeneration in vivo compared to $\mathrm{CPC}$ control without cell delivery. The cell-encapsulating-CPC 
constructs accelerated the resorption of the scaffold in vivo, with CPC-BMP2-iPSMSC having remaining scaffold material that was 7-fold less than CPC control. The novel constructs based on injectable and load-bearing iPSMSC-microbeads in CPC are expected to have wide orthopedic, dental and craniofacial applications to enhance bone regeneration.

\section{Acknowledgments}

We thank Drs. Laurence C. Chow and David J. Mooney for discussions. We thank Dr. Linzhao Cheng for generously providing the hiPSC BC1 cell line. This study was supported by NIH R01 DE14190 and R21 DE22625 (HX), National Science Foundation of China 81401794 (PW), 31100695 and 31328008 (LZ), and University of Maryland School of Dentistry. This paper was presented (PW) as an oral presentation at the 2015 IADR meeting in Boston, and won the IADR Arthur R. Frechette Prosthodontic Research Award.

\section{Disclaimer}

Certain commercial materials and equipment are identified to specify the experimental procedure. This does not imply recommendation or endorsement by NIST or that the material or equipment identified is necessarily the best available for the purpose. Official contribution of the National Institute of Standards and Technology (NIST); not subject to copyright in the United States.

\section{References}

[1] Takahashi K, Yamanaka S. Induction of pluripotent stem cells from mouse embryonic and adult fibroblast cultures by defined factors. Cell 2006;126:663-76.

[2] Kim D, Kim CH, Moon JI, Chung YG, Chang MY, Han BS, et al. Generation of human 
induced pluripotent stem cells by direct delivery of reprogramming proteins. Cell Stem Cell 2009;4:472-6.

[3] Warren L, Manos PD, Ahfeldt T, Loh YH, Li H, Lau F, et al. Highly efficient reprogramming to pluripotency and directed differentiation of human cells with synthetic modified mRNA. Cell Stem Cell 2010;7:618-30.

[4] Hayashi T, Misawa H, Nakahara H, Noguchi H, Yoshida A, Kobayashi N, et al. Transplantation of osteogenically differentiated mouse iPS cells for bone repair. Cell Transplant 2012;21:591-600.

[5] Pagni G, Kaigler D, Rasperini G, Avila-Ortiz G, Bartel R, Giannobile WV. Bone repair cells for craniofacial regeneration. Adv Drug Deliv Rev 2012;64:1310-9.

[6] Lian Q, Zhang Y, Zhang J, Zhang HK, Wu X, Zhang Y, et al. Functional mesenchymal stem cells derived from human induced pluripotent stem cells attenuate limb ischemia in mice. Circulation 2010;121:1113-23.

[7] Zhao Q, Gregory CA, Lee RH, Reger RL, Qin L, Hai B, et al. MSCs derived from iPSCs with a modified protocol are tumor-tropic but have much less potential to promote tumors than bone marrow MSCs. Proc Natl Acad Sci U S A 2015;112:530-5.

[8] Villa-Diaz LG, Brown SE, Liu Y, Ross AM, Lahann J, Parent JM, et al. Derivation of mesenchymal stem cells from human induced pluripotent stem cells cultured on synthetic substrates. Stem Cells 2012;30:1174-81.

[9] Wei H, Tan G, Manasi, Qiu S, Kong G, Yong P, et al. One-step derivation of cardiomyocytes and mesenchymal stem cells from human pluripotent stem cells. Stem Cell Res 2012;9:87-100.

[10] Ko KI, Coimbra LS, Tian C, Alblowi J, Kayal RA, Einhorn TA, et al. Diabetes reduces 
mesenchymal stem cells in fracture healing through a TNFalpha-mediated mechanism. Diabetologia 2015.

[11] Bohner M. Design of ceramic-based cements and putties for bone graft substitution. Eur Cell Mater 2010;20:1-12.

[12] Ginebra MP, Espanol M, Montufar EB, Perez RA, Mestres G. New processing approaches in calcium phosphate cements and their applications in regenerative medicine. Acta Biomater 2010;6:2863-73.

[13] Zhang J, Liu W, Schnitzler V, Tancret F, Bouler JM. Calcium phosphate cements for bone substitution: chemistry, handling and mechanical properties. Acta Biomater 2014;10:1035-49.

[14] Ginebra MP, Canal C, Espanol M, Pastorino D, Montufar EB. Calcium phosphate cements as drug delivery materials. Adv Drug Deliv Rev 2012;64:1090-110.

[15] Bohner M, Gbureck U, Barralet JE. Technological issues for the development of more efficient calcium phosphate bone cements: a critical assessment. Biomaterials 2005;26:6423-9.

[16] Jansen J, Ooms E, Verdonschot N, Wolke J. Injectable calcium phosphate cement for bone repair and implant fixation. Orthop Clin North Am 2005;36:89-95, vii.

[17] Chow LC. Next generation calcium phosphate-based biomaterials. Dent Mater J 2009;28:1-10.

[18] Friedman CD, Costantino PD, Takagi S, Chow LC. BoneSource hydroxyapatite cement: a novel biomaterial for craniofacial skeletal tissue engineering and reconstruction. $\mathbf{J}$ Biomed Mater Res (Appl Biomater) 1998;43:428-32.

[19] Zhou H, Weir MD, Xu HH. Effect of cell seeding density on proliferation and 
osteodifferentiation of umbilical cord stem cells on calcium phosphate cement-fiber scaffold. Tissue Eng Part A 2011;17:2603-13.

[20] Weir MD, Xu HH. Osteoblastic induction on calcium phosphate cement-chitosan constructs for bone tissue engineering. J Biomed Mater Res A 2010;94:223-33.

[21] Tang M, Weir MD, Xu HH. Mannitol-containing macroporous calcium phosphate cement encapsulating human umbilical cord stem cells. J Tissue Eng Regen Med 2012;6:214-24.

[22] Chen W, Zhou H, Tang M, Weir MD, Bao C, Xu HH. Gas-foaming calcium phosphate cement scaffold encapsulating human umbilical cord stem cells. Tissue Eng Part A 2012;18:816-27.

[23] Weir MD, Xu HH. Human bone marrow stem cell-encapsulating calcium phosphate scaffolds for bone repair. Acta Biomater 2010;6:4118-26.

[24] Thein-Han W, Liu J, Xu HH. Calcium phosphate cement with biofunctional agents and stem cell seeding for dental and craniofacial bone repair. Dent Mater 2012;28:1059-70.

[25] Wang P, Liu X, Zhao L, Weir MD, Sun J, Chen W, et al. Bone tissue engineering via human induced pluripotent, umbilical cord and bone marrow mesenchymal stem cells in rat cranium. Acta Biomater 2015.

[26] Villalona GA, Udelsman B, Duncan DR, McGillicuddy E, Sawh-Martinez RF, Hibino N, et al. Cell-seeding techniques in vascular tissue engineering. Tissue Eng Part B Rev 2010;16:341-50.

[27] Zhao L, Weir MD, Xu HH. An injectable calcium phosphate-alginate hydrogel-umbilical cord mesenchymal stem cell paste for bone tissue engineering. Biomaterials 2010;31:6502-10.

[28] Bidarra SJ, Barrias CC, Granja PL. Injectable alginate hydrogels for cell delivery in tissue 
engineering. Acta Biomater 2014;10: 1646-62.

[29] Zhou $\mathrm{H}, \mathrm{Xu} \mathrm{HH}$. The fast release of stem cells from alginate-fibrin microbeads in injectable scaffolds for bone tissue engineering. Biomaterials 2011;32:7503-13.

[30] Chou BK, Mali P, Huang X, Ye Z, Dowey SN, Resar LM, et al. Efficient human iPS cell derivation by a non-integrating plasmid from blood cells with unique epigenetic and gene expression signatures. Cell Res 2011;21:518-29.

[31] Tang M, Chen W, Liu J, Weir MD, Cheng L, Xu HH. Human induced pluripotent stem cell-derived mesenchymal stem cell seeding on calcium phosphate scaffold for bone regeneration. Tissue Eng Part A 2014;20:1295-305.

[32] Liu J, Chen W, Zhao Z, Xu HH. Reprogramming of mesenchymal stem cells derived from iPSCs seeded on biofunctionalized calcium phosphate scaffold for bone engineering. Biomaterials 2013;34: 7862-72.

[33] Chen W, Thein-Han W, Weir MD, Chen Q, Xu HH. Prevascularization of biofunctional calcium phosphate cement for dental and craniofacial repairs. Dent Mater 2014;30:53544.

[34] Lee K, Weir MD, Lippens E, Mehta M, Wang P, Duda GN, et al. Bone regeneration via novel macroporous CPC scaffolds in critical-sized cranial defects in rats. Dent Mater 2014;30:e199-207.

[35] Ji W, Yang F, Ma J, Bouma MJ, Boerman OC, Chen Z, et al. Incorporation of stromal cell-derived factor-1alpha in PCL/gelatin electrospun membranes for guided bone regeneration. Biomaterials 2013;34:735-45.

[36] Pilipchuk SP, Plonka AB, Monje A, Taut AD, Lanis A, Kang B, Giannobile WV. Tissue engineering for bone regeneration and osseointegration in the oral cavity. Dent Mater 
2015.

[37] Rosa V, Della Bona A, Cavalcanti BN, Nor JE. Tissue engineering: from research to dental clinics. Dent Mater 2012;28:341-8.

[38] Mao JJ, Giannobile WV, Helms JA, Hollister SJ, Krebsbach PH, Longaker MT, et al. Craniofacial tissue engineering by stem cells. J Dent Res 2006;85:966-79.

[39] Bianco P, Cao X, Frenette PS, Mao JJ, Robey PG, Simmons PJ, et al. The meaning, the sense and the significance: translating the science of mesenchymal stem cells into medicine. Nat Med 2013;19:35-42.

[40] Chen YS, Pelekanos RA, Ellis RL, Horne R, Wolvetang EJ, Fisk NM. Small molecule mesengenic induction of human induced pluripotent stem cells to generate mesenchymal stem/stromal cells. Stem Cells Transl Med 2012;1:83-95.

[41] Diederichs S, Tuan RS. Functional comparison of human-induced pluripotent stem cellderived mesenchymal cells and bone marrow-derived mesenchymal stromal cells from the same donor. Stem Cells Dev 2014;23:1594-610.

[42] Hynes K, Menicanin D, Han J, Marino V, Mrozik K, Gronthos S, et al. Mesenchymal stem cells from iPS cells facilitate periodontal regeneration. J Dent Res 2013;92:833-9.

[43] Hynes K, Menicanin D, Mrozik K, Gronthos S, Bartold PM. Generation of functional mesenchymal stem cells from different induced pluripotent stem cell lines. Stem Cells Dev 2014;23:1084-96.

[44] Kang H, Shih YR, Hwang Y, Wen C, Rao V, Seo T, et al. Mineralized gelatin methacrylate-based matrices induce osteogenic differentiation of human induced pluripotent stem cells. Acta Biomater 2014;10:4961-70.

[45] Brown SE, Tong W, Krebsbach PH. The derivation of mesenchymal stem cells from 
human embryonic stem cells. Cells Tissues Organs 2009;189:256-60.

[46] Boyd NL, Robbins KR, Dhara SK, West FD, Stice SL. Human embryonic stem cellderived mesoderm-like epithelium transitions to mesenchymal progenitor cells. Tissue Eng Part A 2009;15:1897-907.

[47] Illich DJ, Demir N, Stojkovic M, Scheer M, Rothamel D, Neugebauer J, et al. Concise review: induced pluripotent stem cells and lineage reprogramming: prospects for bone regeneration. Stem Cells 2011;29:555-63.

[48] Li F, Niyibizi C. Cells derived from murine induced pluripotent stem cells (iPSC) by treatment with members of TGF-beta family give rise to osteoblasts differentiation and form bone in vivo. BMC Cell Biol 2012;13: 35.

[49] Levi B, Hyun JS, Montoro DT, Lo DD, Chan CK, Hu S, et al. In vivo directed differentiation of pluripotent stem cells for skeletal regeneration. Proc Natl Acad Sci U S A 2012;109:20379-84.

[50] de Peppo GM, Marcos-Campos I, Kahler DJ, Alsalman D, Shang L, Vunjak-Novakovic G, et al. Engineering bone tissue substitutes from human induced pluripotent stem cells. Proc Natl Acad Sci U S A 2013;110:8680-5.

[51] Vo TN, Kasper FK, Mikos AG. Strategies for controlled delivery of growth factors and cells for bone regeneration. Adv Drug Deliv Rev 2012;64:1292-309.

[52] $\mathrm{Xu} \mathrm{HH}$, Weir MD, Simon CG. Injectable and strong nano-apatite scaffolds for cell/growth factor delivery and bone regeneration. Dent Mater 2008;24:1212-22.

[53] Lee KY, Mooney DJ. Alginate: properties and biomedical applications. Prog Polym Sci 2012;37:106-126.

[54] Qiao P, Wang J, Xie Q, Li F, Dong L, Xu T. Injectable calcium phosphate-alginate- 
chitosan microencapsulated MC3T3-E1 cell paste for bone tissue engineering in vivo. Mater Sci Eng C Mater Biol Appl 2013;33: 4633-9.

[55] Zou D, Guo L, Lu J, Zhang X, Wei J, Liu C, et al. Engineering of bone using porous calcium phosphate cement and bone marrow stromal cells for maxillary sinus augmentation with simultaneous implant placement in goats. Tissue Eng Part A 2012;18:1464-78.

[56] Mastrogiacomo M, Papadimitropoulos A, Cedola A, Peyrin F, Giannoni P, Pearce SG, et al. Engineering of bone using bone marrow stromal cells and a silicon-stabilized tricalcium phosphate bioceramic: evidence for a coupling between bone formation and scaffold resorption. Biomaterials 2007;28:1376-84.

\section{Figure captions}

[1] Cell encapsulation and release from the microbeads (A-D): at $3 \mathrm{~d}$, iPSMSCs started to be released from microbeads; from 7 to $14 \mathrm{~d}$, more cells were released and the contour of microbeads became obscure as alginate degraded. Cell viability and proliferation of iPSMSC-microbeads (E-L): live/dead staining and CCK-8 from 1 to $14 \mathrm{~d}$ showed good cell viability and proliferation, and cell viability (absorbance at $450 \mathrm{~nm}$ ) was not negatively affected by injection (K). CCK-8 cell viability (absorbance at $450 \mathrm{~nm}$ ) increased with culture time $(\mathrm{L})$. Each value is mean $\pm \mathrm{SD}(\mathrm{n}=6)$. Bars with dissimilar letters indicate significantly different values $(\mathrm{p}<0.05)$.

[2] Osteogenic differentiation and mineralization of the encapsulated cells in OS media. Folds of change in gene expressions for ALP (A), Runx2 (B), COL1A1 (C), and OCN (D) were plotted. The Ct value of iPSMSCs in growth media on TCPS for $1 \mathrm{~d}$ served as the 
calibrator. Mineral synthesis by cells was detected by Alizarin Red staining (E). The bone mineral matrix became denser and darker red with increasing time. Cell-synthesized mineral concentration was measured by the osteogenesis assay $(\mathrm{F})$. Each value is mean \pm $\operatorname{SD}(n=5)$. Bars with dissimilar letters indicate significantly different values $(\mathrm{p}<0.05)$.

[3] Osteogenic differentiation of BMSCs when co-cultured with cell-encapsulatingmicrobeads in the absence of OS media. Folds of change in gene expressions for ALP (A), Runx2 (B), COL1A1 (C), and OCN (D) were plotted. The Ct value of BMSCs in growth media cultured on TCPS for 1d served as the calibrator. Osteogenic markers were upregulated in all groups, indicating that active factors were released from the encapsulated cells and acted on the BMSCs. Each value is mean $\pm \operatorname{SD}(n=5)$. Bars with dissimilar letters indicate significantly different values $(\mathrm{p}<0.05)$.

[4] Histological observations of bone regeneration in cranial bone defects in rats: HE and MT staining. The periosteal side was on the top, while the dura side was at the bottom. In HE, bone tissue was stained in red and residual materials (mainly CPC) were stained in purple or pink. In MT, the bone tissue was stained in deep blue or red, the residual materials were stained in light blue or white, and the collagen fibers were blue-stained fibrous structures. (I) and (J) were high magnification images of typical new bone structure within the defects of the dotted-line rectangle areas in $(\mathrm{E})$ and $(\mathrm{F})$, respectively.

[5] IHC staining of human nuclear antigen $12 \mathrm{w}$ after implantation (A-D). The insets were negative control without primary antibody incubation. Positive staining (marked by arrows) was mainly found within the non-mineralized fibrous tissue in the cellencapsulated scaffolds. There were no positive areas in the CPC control. Histomorphometry of the new bone area fraction (E) and residual material area fraction 
(F). Bars with dissimilar letters indicate significantly different values $(\mathrm{p}<0.05)$. Each value is mean $\pm \operatorname{SD}(\mathrm{n}=5)$. 

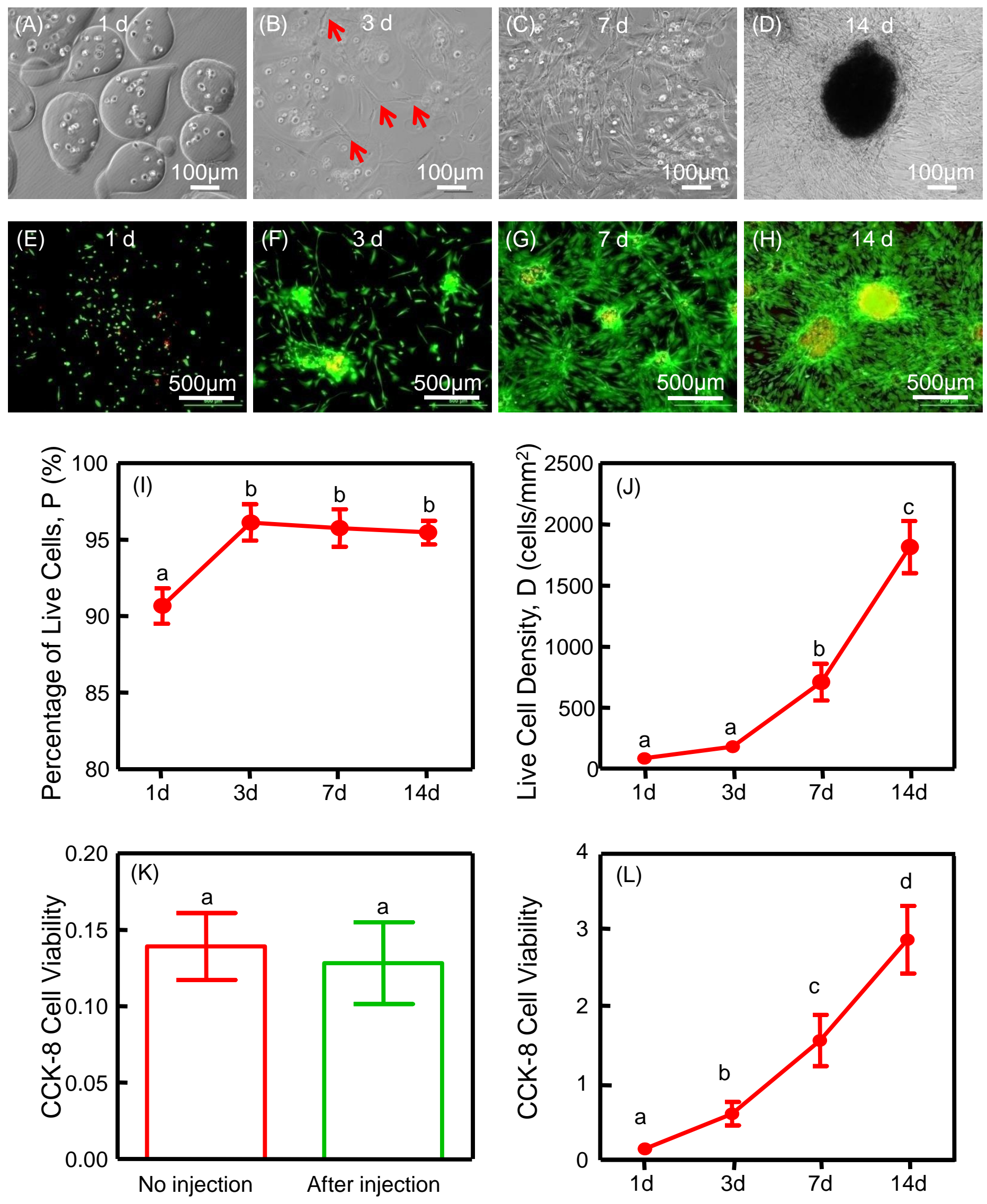

Fig. 1 

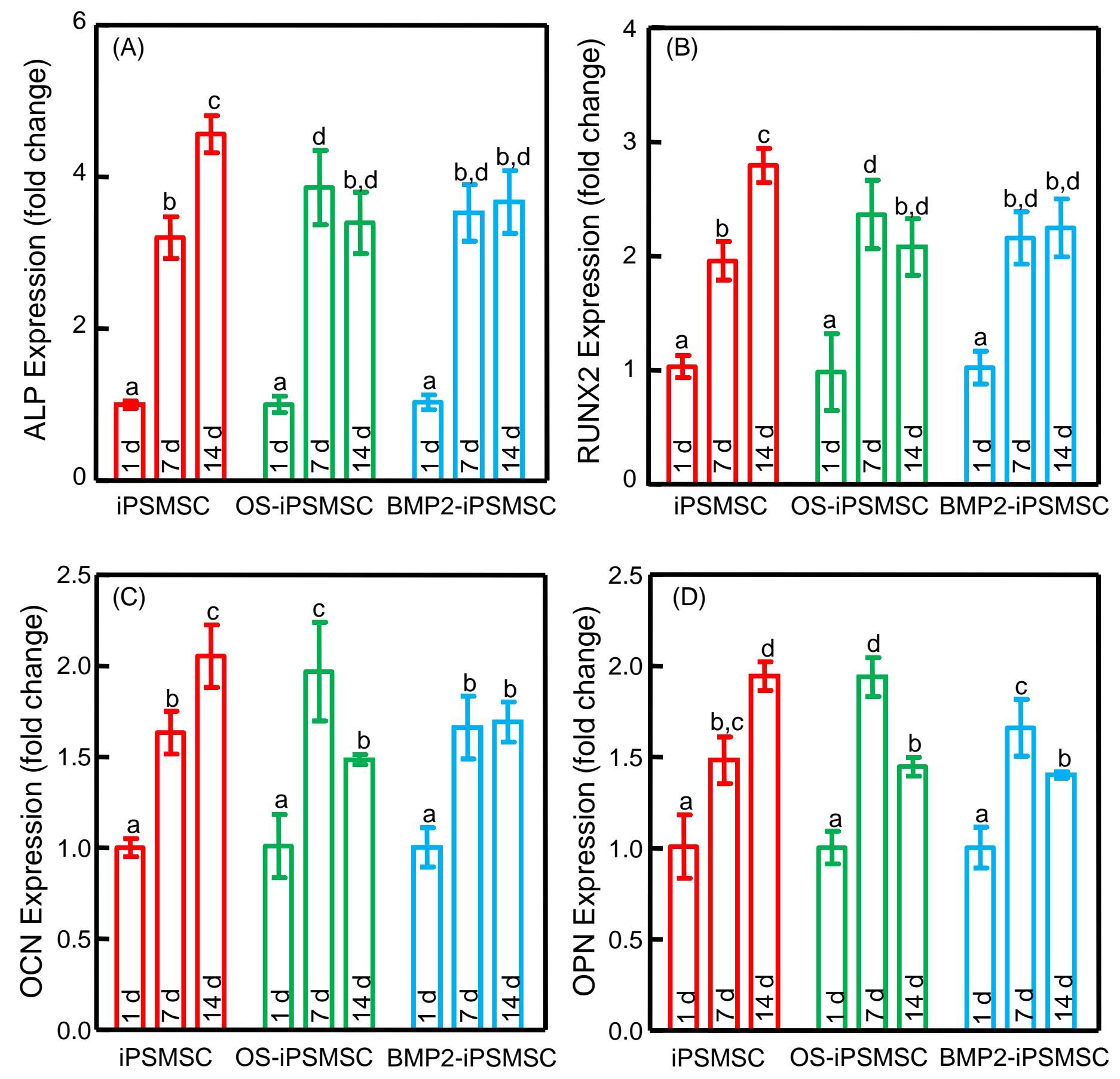

Fig. 3 


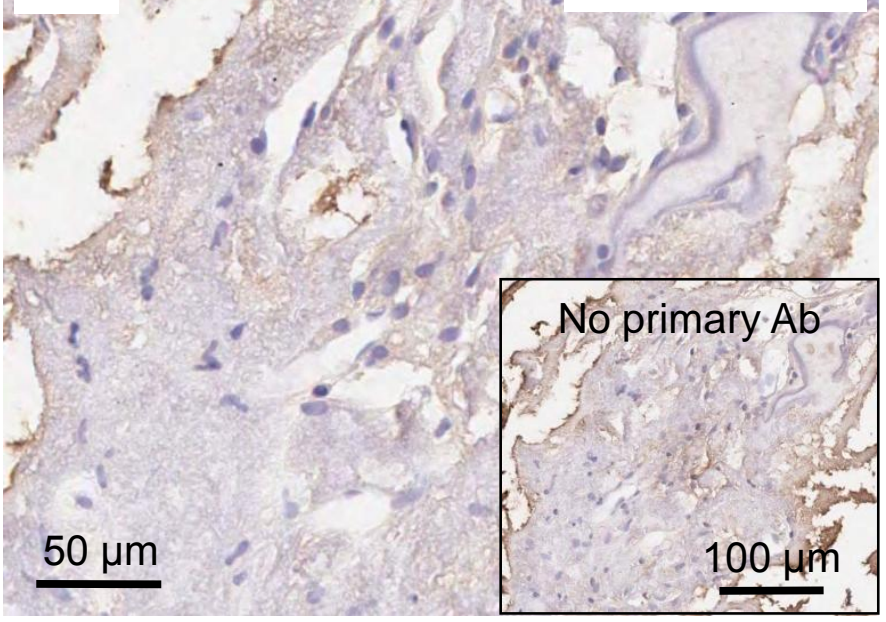

(C)

(C) $\frac{1}{2}+4$ CPC-OS-iPSMSCs
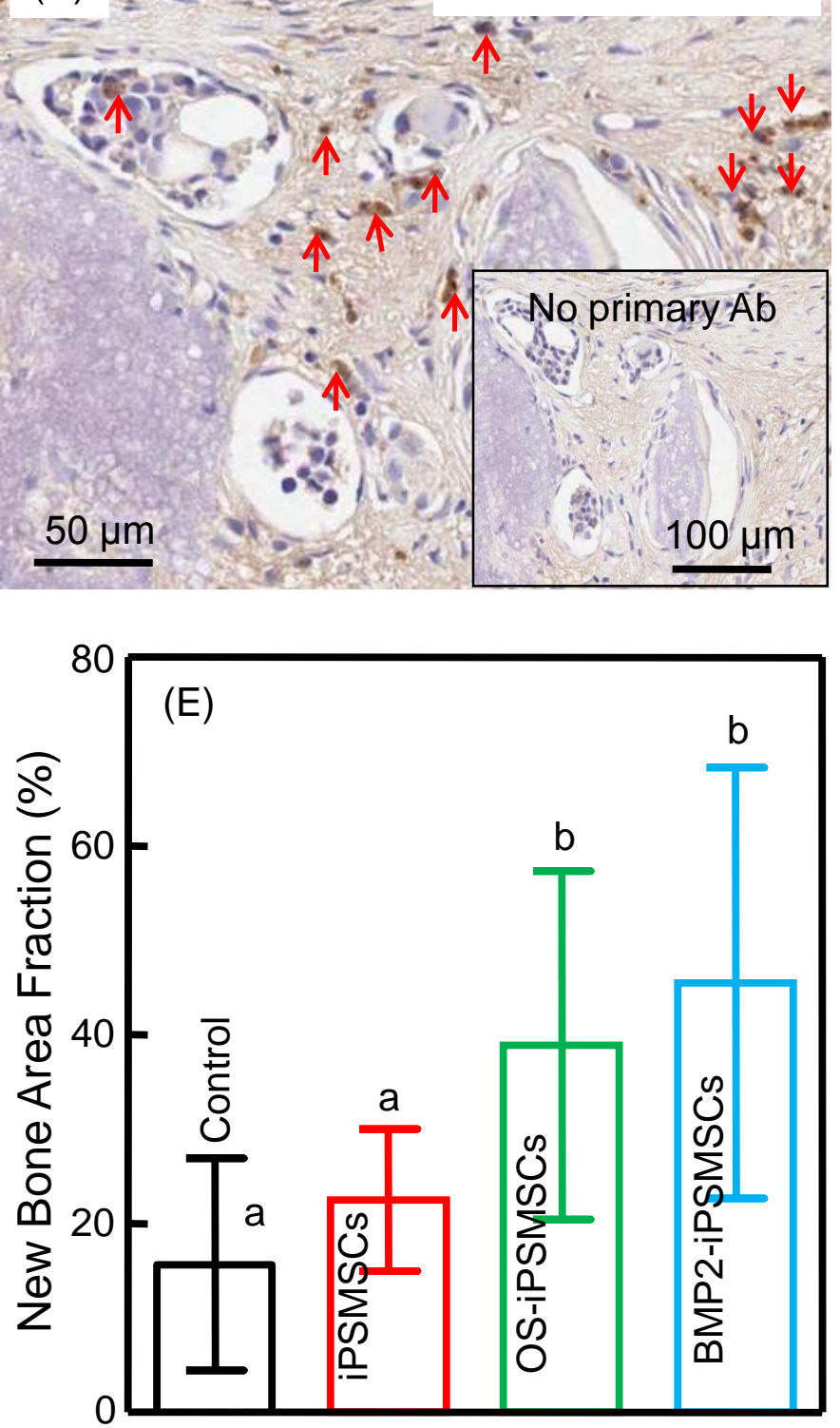

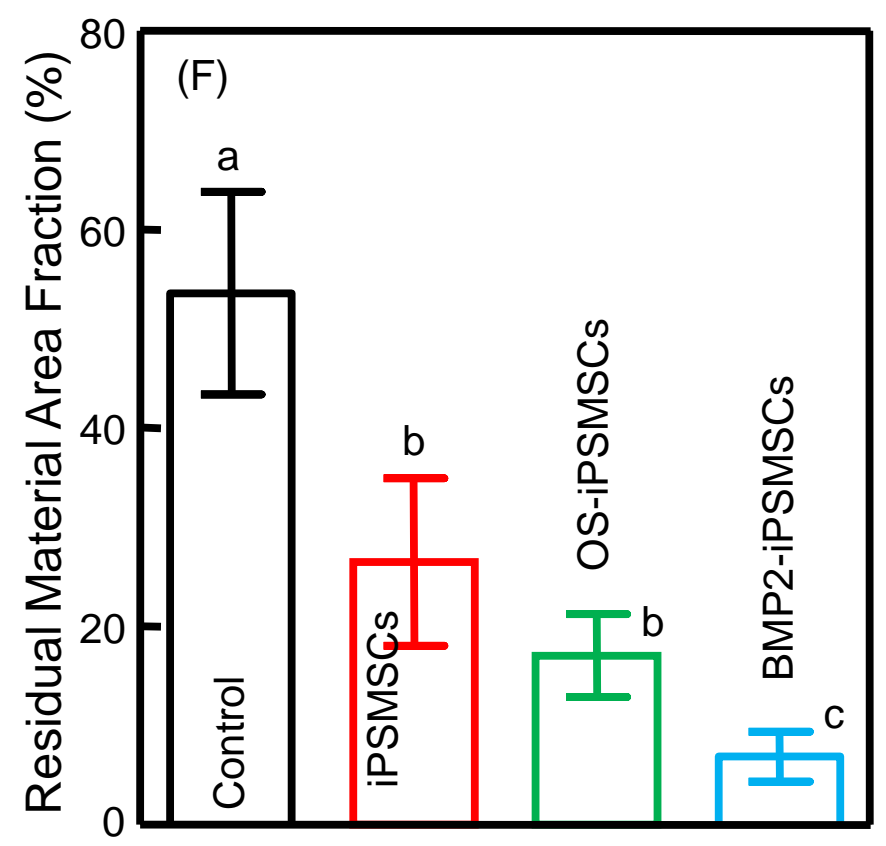

Fig. 5

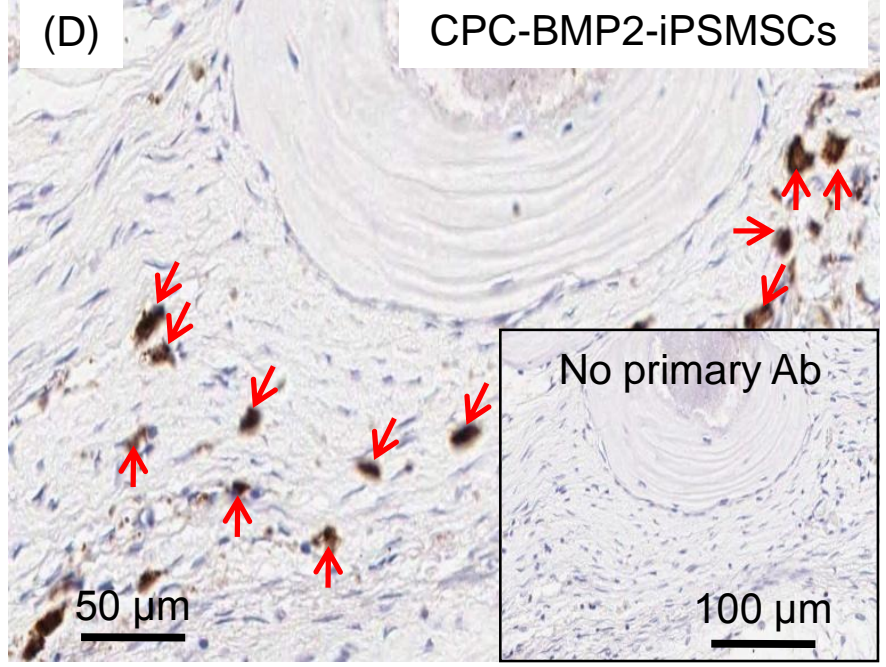

